\title{
Sensor impedimétrico para la detección de bacterias patogénicas mediante péptidos antimicrobianos
}

Accésit Congreso SIBB 2015

\author{
M. HOYOS-NOGUÉS $1,2,{ }^{*}$, S. BROSEL-OLIU ${ }^{3}$, N. ABRAMOVA ${ }^{3}$, \\ A. BRATOV ${ }^{3}$, C.MAS-MORUNO ${ }^{1,2}$, F.J GIL ${ }^{1,2}$ \\ ${ }^{1}$ Biomaterials, Biomechanics and Tissue Engineering Group, Technical University of Catalonia, Spain \\ ${ }^{2}$ Centre for Research in Nano Engineering, Technical University of Catalonia, Spain \\ ${ }^{3}$ BioMEMS Group, Centre Nacional de Microelectrònica (IMB-CNM, CSIC), Spain
}

\begin{abstract}
Resumen
La peri-implantitis, una inflamación causada por la formación del biofilm, es una de las causas más importantes de la fallida de los implantes en odontología [1]. Por esto, la detección de bacterias patogénicas al inicio del proceso de formación de biofilms, representa una estrategia muy potente para la prevención de las infecciones en los implantes. Entre los diferentes métodos para la detección de bacterias patogénicas, los biosensores electroquímicos, especialmente los sistemas basados en impedancia (EIS), presentan una serie de ventajas como la miniaturización, la mejora en sensibilidad y el bajo coste. En este sentido, los péptidos antimicrobianos (AMPS), conocidos como componentes del sistema inmune y con actividad hacia las bacterias, pueden ser usados para desarrollar elementos de bioreconocimiento altamente efectivos. Por lo tanto, el objetivo de este estudio es la combinación del uso de EIS y la habilidad de los AMPs para obtener biosensores con alta sensibilidad, especificidad y límites de detección muy bajos para la detección de bacterias patogénicas.
\end{abstract}

Palabras clave: Biosensor impedimétrico, peri-implantitis, péptidos antimicrobianos (AMPs).

\begin{abstract}
Peri-implantitis, an inflammation caused by biofilm formation, constitutes a major cause of implant failure in dentistry [1]. Thus, the detection of bacterial pathogens at the early steps of biofilm growth represents a very powerful strategy to prevent from implant-related infections. Among different methods to detect pathogenic bacteria, electrochemical biosensors, especially impedance-based systems (EIS), present key advantages in terms of miniaturization, improvement in sensitivity and low cost. In this regard, antimicrobial peptides (AMPS), which are well-known components of the immune system and active towards bacteria, can be used to develop highly effective biological recognition elements. Thus, the aim of the present study is to combine the use of EIS and the ability of AMPs to obtain biosensors with high sensitivity, specificity and low detection limits for pathogenic bacteria.
\end{abstract}

Keywords: Impedimetric biosensor, peri-implantitis, antimicrobial peptides (AMPs).

Correspondencia:

Mireia Hoyos Nogués

Email: mireia.hoyos@upc.edu 


\section{Introducción}

Los implantes dentales son hoy en día uno de los recursos más frecuentes usados como sustitutos artificiales de la raíz de un diente perdido. Aunque los casos de éxito de un implante son elevados, existe aún un porcentaje muy significativo de fracaso de implantes [2]. Una de las partes más criticas de implante es el surco gingival, que es el punto de unión entre la mucosa gingival y el cuello del implante. La integridad de este sellado biológico gingival, es de gran interés debido a los posibles daños asociados con un sellado de calidad inferior: la invasión bacteriana y subsecuente colonización derivando en enfermedades como la peri-implantitis. La peri-implantitis se define como un proceso inflamatorio de la mucosa que rodea al implante provocando la pérdida del soporte óseo y es una de las principales causas de fracaso del implante. Su aparición requiere un diagnóstico y tratamiento precoz para evitar el fracaso del implante. Para ello es necesario entender cuál es el desarrollo de la adhesión bacteriana y como se comportan estas especies.

La peri-implantitis no es el resultado de la infección por una sola cepa de bacterias, sino es fruto de un complejo ecosistema dinámico de bacterias conocido como placa dentobacteriana (o biofilm). El proceso de formación de este biofilm está formado por varias etapas [3]: en una primera fase, la presencia de nutrientes y glicoproteínas promueve la adhesión de los llamados colonizadores primarios, tales como el Streptococcus gordonii. Esta primera capa de bacterias inicia una cadena de señalización produciendo la adhesión de las bacterias de transición, como el Streptococcus sanguinis. Estas, a su vez, promueven la adhesión de los colonizadores secundarios, donde encontra-mos las especies más periodontopatogénicas, como el Porphyromonas gingivalis [4]. Poco a poco las bacterias se acomodan con la ayuda de los pili y se va generando una red de bacterias más estructurada. Finalmente, con la secreción de una matriz extracelular de las propias bacterias, se origina el biofilm, una es-tructura compleja y altamente resistente a los tratamientos antibióticos [5].
Para evitar el desarrollo del biofilm, necesitamos realizar una detección precoz. Los métodos convencionales para la detección e identificación bacteriana están basados principalmente en técnicas de laboratorio, como el cultivo celular, análisis microscópico y ensayos bioquímicos que conllevan largos tiempos de procesado e implementación y una baja sensibilidad [6]. Con el objetivo de superar estas restricciones, se necesitan nuevos métodos y entre ellos, los biosensores son unos de los más prometedores. Recientemente, se han realizado investigaciones muy interesantes en biosensores para la detección de bacterias patogénicas [7].

Los biosensores se constituyen principalmente de dos elementos: una unidad de bioreconocimiento, que es la parte responsable de detectar la sustancia que queremos analizar; y el transductor, el cual convierte la interacción entre nuestra muestra y la primera unidad funcional y la convierte en una señal medible y procesable.

Los biosensores pueden clasificarse dependiendo del tipo de elemento de reconocimiento que usan. Hasta ahora los biosensores para detectar microrganismos patógenos han sido principalmente inmunosensores. Sin embargo, la inestabilidad en condiciones ambientales extremas y la detección de un solo analito, limita la aplicación práctica de estos sistemas. Los basados en ácidos nucleicos, requieren una extracción eficiente del DNA y algunos incluso una amplificación mediante PCR. Por el contrario, la facilidad en la síntesis, la buena estabilidad y el amplio espectro hacia una gran variedad de microrganismos, hacen que los péptidos antimicrobianos (AMPs) sean unos candidatos interesantes para su uso como elementos de reconocimiento [8].

Los biosensores también se pueden categorizar según la señal de transducción: puede ser mecánica, óptica o eléctrica. Hay un especial interés en los biosensores electroquímicos, sobretodo los basados en impedancia (EIS), ya que presentan importantes ventajas en la facilidad de miniaturización, el no uso de reactivos, la alta sensibilidad y un bajo coste [6].

Una vez vistas todas las premisas, podemos establecer el objetivo de nuestro proyecto: 
diseñar un sistema de detección precoz para bacterias mediante el uso de sistemas impedimétricos funcionalizados con AMPs. Todo ello con el propósito de poder evitar el desarrollo de la peri-implantitis y una consecuente caída del implante.

\section{Materiales y métodos}

\section{Diseño del sistema}

Los sensores escogidos son electrodos fabricados y diseñados en el grupo de biomems del CSIC en Bellaterra $[9,10]$. El tipo de sensores utilizados son sensores interdigitados fabricados con tántalo de silicio basados en impedancia (EIS). Partimos de dos morfologías: una planar (IDEA) y una que contiene unas barreras de dióxido de silicio de $4 \mu \mathrm{m}$ de altura entre los dedos interdigitados (3D-IDEA). Ambas morfologías se pueden ver en las Figuras $1 \mathrm{~A}$ (IDEA planar) y $1 \mathrm{~B}$ (3D-IDEA).
En cuanto al elemento de bioreconocimiento de nuestro sensor, ya hemos comentado que usaremos los péptidos antimicrobianos (AMPs). Los péptidos cargados positivamente, como la lactoferrina, se sienten atraídos electrostáticamente por la membrana celular cargada negativamente de las bacterias. Esta habilidad para interaccionar con las bacterias permite el uso de los AMPs en sistemas de detección. El AMP escogido es un péptido derivado de la lactoferrina humana, el hFL1-11, catiónico e hidrofóbico, y que se ha sintetizado en nuestro grupo y demostrada su actividad bactericida [11]. La estructura química se puede ver en la Figura 1C.

\section{Optimización de la biofuncionalización}

La técnica usada para inmovilizar el AMP en la superficie del sensor es la silanización. Un primer paso ha sido optimizar este proceso, probando dos métodos: silanización en gas

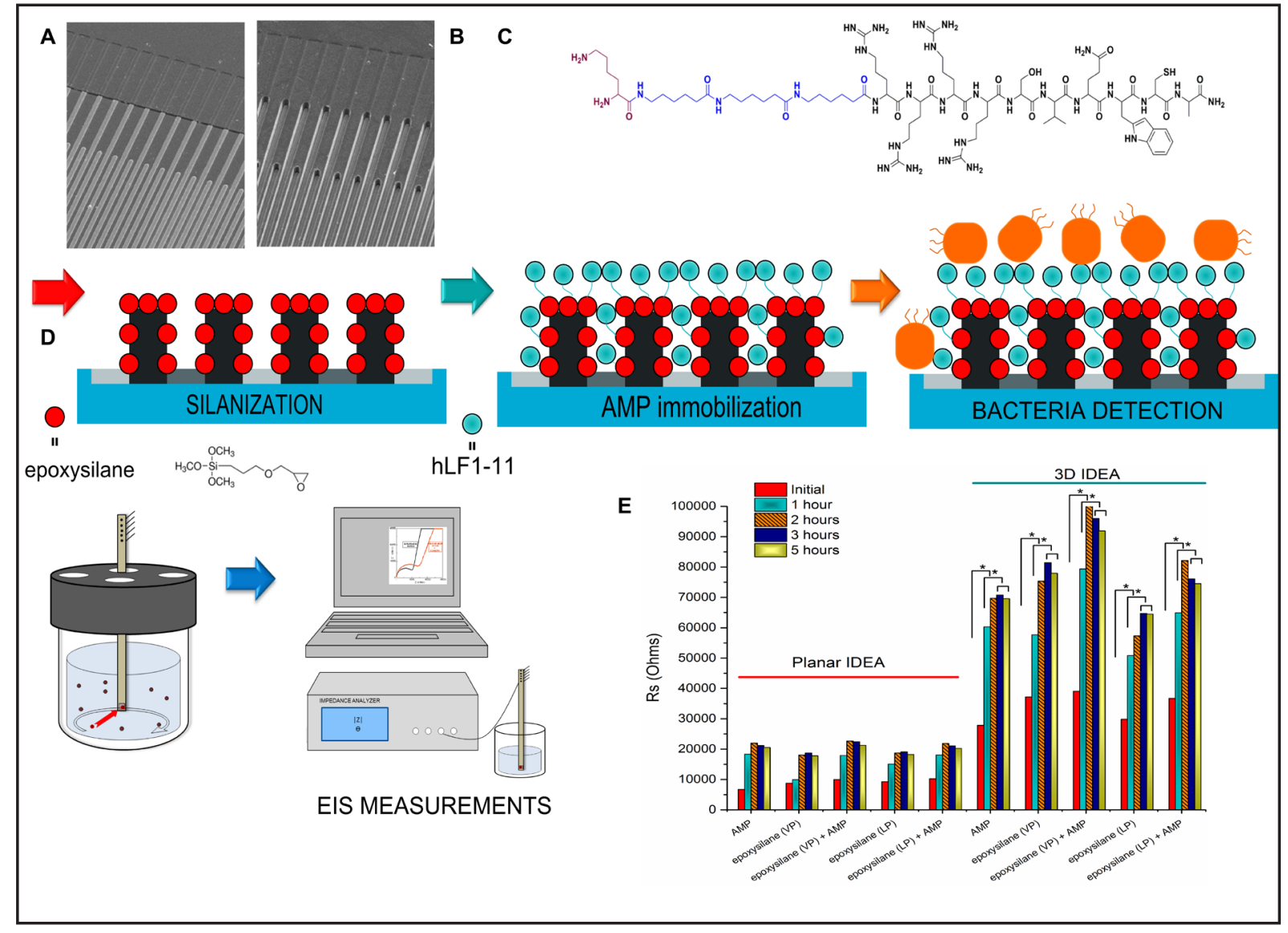

Figura 1. (A) Imagen SEM de la morfología planar. (B) Imagen SEM de la morfología 3D (3DIDEA). (C) Estructura química de la lactoferrina ( $h L f 1-11)$. (D) Proceso de biofuncionalización del sensor. (E) Optimización de la biofuncionalización mediante EIS. Optimización entre diferentes geometrías del sensor (planar y 3D) y entre diferentes métodos de silanización (gas-VP y líquida-LP). 
(VP) durante 1 hora en atmosfera controlada a $50^{\circ} \mathrm{C}$ con (3-glycidyloxypropyl) trimethoxysilane (epoxisilano); y silanización en líquido (LP), que consistió en sumergir los electrodos en una solución de epoxisilano y ethanol absoluto $(1: 10, v / v)$ durante 1 hora. Posteriormente, se sumergió el electrodo en una solución del péptido a $100 \mu \mathrm{M}$ con PBS a pH neutro y a pH básico (8.5). Se probaron todos los métodos tanto con los IDEA planar como con los 3D-IDEA. El proceso de biofuncionalización está esquematizado en la Figura 1D.

\section{Detección bacteriana}

Para todos los ensayos, los sensores han sido incubados con cultivos bacterianos de Streptococcus sanguinis, como modelo de bacteria oral. S. sanguinis se cultivaron en caldo de cultivo Todd-Hewitt (TH) y se dejó crecer toda la noche a $37^{\circ} \mathrm{C}$ antes de cada ensayo. Se ajustó mediante densidad óptica la concentración bacteriana para obtener $10^{8} \mathrm{CFU} \bullet \mathrm{mL}^{-1}$.

Después de los tiempos de incubación se han ido tomando medidas impedimétricas en soluciones de KCL $10^{-5} \mathrm{M}$ con la conductividad controlada en todo momento. El equipo usado fue el Quadtech 7600 Plus LCR Meter. Para el análisis de los resultados se usó el software de Z-Plot/Z-View.

\section{Ensayo de citotoxicidad}

Fibroblastos humanos (hFFs) fueron cultivados en medio Dulbecco Eagle's enriquecido con un $10 \%(\mathrm{v} / \mathrm{v})$ de fetal bovine serum (FBS), $1 \%(\mathrm{w} / \mathrm{v})$ de L-glutamine y $1 \%$ de penicillin/ streptomycin $\left(50 \mathrm{U} \bullet \mathrm{mL}^{-1}\right.$ y $\left.50 \mathrm{mg} \bullet \mathrm{m} \mathrm{L}^{-1}\right)$.

Las muestras control y los 3D-IDEA funcionalizados con el AMP se usaron para realizar el ensayo de citotoxicidad indirecta con hFFs. Este ensayo estuvo basado en la medida de la liberación del enzima de lactate dehydrogenase $(\mathrm{LDH})$ mediante el Kit de Detección de Citotoxicidad LDH.

\section{Límite de detección}

Con el fin de calcular la sensibilidad de los biosensores, se realizaron medidas de impedancia en $\mathrm{KCl}$ y en una solución de saliva artificial $[12,13]$. Se estableció un rango de diferentes concentraciones de $S$. sanguinis: desde
$10^{1}$ hasta $10^{6} \mathrm{CFU} \cdot \mathrm{mL}^{-1}$ en condiciones dinámicas para poder garantizar una distribución homogénea de las bacterias en el volumen total. Los electrodos fueron sumergidos en la solución $1 \mathrm{~h}$ para cada una de las concentraciones y se realizaron medidas de impedancia para cada electrodo.

\section{Análisis estadístico}

Las diferencias significativas entre los diferentes grupos fueron analizadas mediante el test paramétrico ANOVA seguido de un test comparativo de Tukey para minimizar el error. También se usó el test no paramétrico de Kruskall-Wallis para verificar los resultados. Los niveles de significancia se establecieron al 95\%.

\section{Resultados y discusión}

\section{Optimización de la biofuncionalización}

Tal y como se muestra en los datos analizados (Figura 1E), se observan diferencias significativas entre las diferentes técnicas de inmovilización del péptido. Los resultados de impedancia indican que la silanización VP permite una mayor impedancia comparado con la silanización LP o bien con los métodos de fisisorción. Esto sugiere que la silanización VP promueve un mayor anclaje del AMP en la superficie de los IDEA. Esto concuerda con estudios anteriores donde se demostraba que la silanización VP era más efectiva que la silanización LP en la adhesión de moléculas [14]. Los resultados también indican que aunque la técnica de fisisorción también da unos buenos resultados de impedancia, la estabilidad de este tipo de unión es inferior a la obtenida con la silanización, la cual ofrece un enlace covalente y estable [15].

Si nos fijamos en la geometría del sensor, la respuesta impedimétrica correspondiente al 3D-IDEA es mayor que el IDEA planar, dando a entender una mayor sensibilidad. Debido a su diseño específico, cuando un potencial se aplica entre los electrodos del 3D-IDEA, la mayor parte del flujo de corriente pasa cerca de la superficie de las barreras originando una microcapilaridad en cada electrodígito. Cambios en la distribución eléctrica de carga 
en la interface sólido/líquido provocan a su vez cambios en la conductividad a lo largo de la superficie de las barreras $[10,16]$. Estos resultados concuerdan con estudios reportados anteriormente $[9,17]$. Todo ello sugiere una mayor sensibilidad para la morfología 3D, con lo que los sensores 3D-IDEA se eligieron para realizar el resto de experimentos. Otro dato que se extrae de la Figura 1E es que después de 3 h de estabilización en $\mathrm{KCl}$, el valor de impedancia se mantuvo invariable en todas las condiciones.

\section{Detección bacteriana}

Tal y como se muestra en la Figura 2A, la presencia de AMP provocó un incremento en la respuesta de Rs después de $1 \mathrm{~h}$ de incubación, comparado con la muestra control. Este aumento fue más pronunciado en la condición del péptido con enlace covalente (silanizado). Este efecto es probablemente debido a la pre- sencia de la unidad de ancoraje y espaciador del péptido hLF1-11, que asegura un enlace covalente de la molécula con el epoxisilano y una accesibilidad del motivo antimicrobial para favorecer la interacción con la bacteria. Además, el estudio sugiere que la presencia del péptido hLF1-11en la superficie del sensor permite registrar la interacción de $S$. sanguinis en las dos primeras horas de incubación. De todas estas observaciones se deriva que estos sistemas basados en sensores de impedan-cia recubiertos con AMP, pueden detectar de manera efectiva la presencia de bacterias en la superficie.

\section{Ensayo de citotoxicidad}

La posibilidad de integrar esta plataforma de detección en un implante dental hace necesario comprobar que los electrodos no son tóxicos para las células humanas. Para ello, se estudió la citotoxicidad indirecta de los elec-

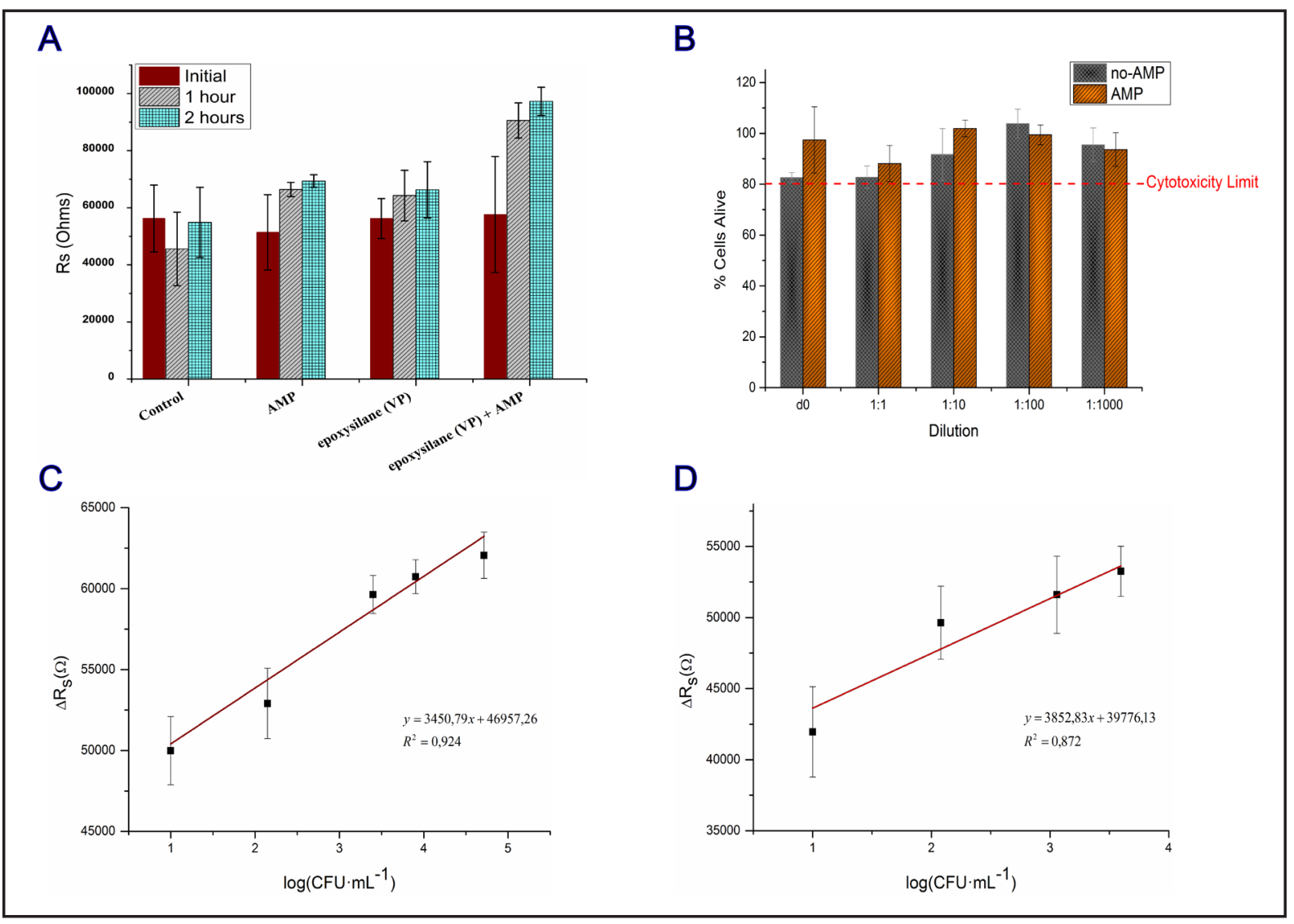

Figura 2. (A) Ensayo de detección en 3D-IDEA. (B) Ensayo de citotoxicidad con hFFs. (C) Regresión lineal de las medidas de impedancia con S. sanguinis en una solución de KCl. (D) Regresión lineal de las medidas de impedancia con S. sanguinis en una solución de saliva artificial. Los electrodos estuvieron en contacto con cada solución $1 \mathrm{~h}$. 
trodos (tanto los iniciales como los funcionalizados con la molécula) con fibroblastos humanos (hFFs). El ensayo se realizó después de 1 día de incubación en diferentes concentraciones de medio previamente en contacto con los electrodos. Los resultados se obtuvieron mediante el Kit de detección de Citotoxicidad (LDH). Los datos obtenidos (Figura 2B) muestran como todas las superficies evaluadas ofrecen unos valores de viabilidad por encima del $80 \%$ para todas las diluciones examinadas. Estos resultados evidencian como ni los sensores ni la molécula de péptido hLF1-11 tienen efectos citotóxicos hacia las células de fibroblasto.

\section{Límite de detección}

Para evaluar la sensibilidad del biosensor desarrollado, se realizaron medidas de impedancia usando $S$. sanguinis en diferentes concentraciones. Tal y como se muestra en la Figura 2C, se establece una correlación lineal entre $\Delta$ Rs y el logaritmo de la concentración bacteriana entre $10^{1}-10^{5} \mathrm{CFU} \cdot \mathrm{mL}^{-1}$ con un coeficiente de correlación de $\mathrm{R}^{2}=0.924$. La ecuación del modelo lineal se puede expresar mediante la ecuación: $y=b x+a$. Este modelo es usado para el cálculo de la sensibilidad del sistema, b, versus el logaritmo de la concentración bacteriana, obteniendo un valor de $3.45 \pm 0.5 \mathrm{k} \Omega$ por década de concentración bacteriana. El límite de detección (LoD), se determina mediante: $\mathrm{LoD}=3 \cdot \mathrm{Sa} / \mathrm{b}$ [18], donde Sa es la desviación estándar y b la pendiente de la curva. El LoD se estableció a $3.5 \bullet 10^{1}$ $\mathrm{CFU} \cdot \mathrm{mL}^{-1}$, el cual es comparable e incluso mejor que otros valores de limite de detección reportados para sistemas de detección de bacterias [19].

Se usó saliva artificial como medio de cultivo para $S$. sanguinis con el fin de simular la salinidad y la conductividad del entorno bucal y validar así el uso del biosensor para detección en condiciones fisiológicas. Se realizaron ensayos de detección como hemos explicado anteriormente pero en el rango entre $10^{1} \mathrm{CFU} \cdot \mathrm{mL}^{-1}$ y $10^{5} \mathrm{CFU} \cdot \mathrm{mL}^{-1}$ y usando saliva artificial en vez de $\mathrm{KCl}$ (Figura 2D). Como ilustra el gráfico, ahora la respuesta sigue una correlación lineal $\left(\mathrm{R}^{2}=0.872\right)$ en el rango de
$10^{1}-10^{4} \mathrm{CFU} \cdot \mathrm{mL}^{-1}$. En este caso la sensibilidad del sensor fue de $3.85 \pm 1.3 \mathrm{k} \Omega$ por década de concentración bacteriana. El LoD se obtuvo de $8.6 \cdot 10^{2} \mathrm{CFU} \cdot \mathrm{mL}^{-1}$. Esta diferencia respecto los resultados obtenido en $\mathrm{KCl}$ es probablemente debido a que el factor saliva puede influenciar en la respuesta del biosensor. De todos modos, el valor obtenido es mejor que los reportados en otros estudios [20].

\section{Conclusiones}

En conclusión, el uso de AMP como elementos de bioreconocimiento en sensores $3 \mathrm{D}$ IDEA, ha permitido la implementación de un sistema de detección rápido y fácil de usar con una elevada sensibilidad, para la detección de la cepa periodontopatógenica S. sanguinis, que ha sido elegida como ejemplo de colonizador primario. Este array integrado y basado en AMP puede ser usado para la detección de un amplio espectro de bacterias y de gran interés para la prevención de la formación inicial de biofilm y las consecuentes infecciones relacionadas.

\section{Agradecimientos}

Los autores agradecen el soporte económico de MINECO (MAT 2012-30706) y del Government of Russian Federation (Grant 074U01).

\section{Referencias}

1. A. Lee, H.-L. Wang, Biofilm related to dental implants., Implant Dent. 19 (2010) 387-93.

2. H. Algraffee, F. Borumandi, L. Cascarini, Peri-implantitis, Br. J. Oral Maxillofac. Surg. 50 (2012) 689-694.

3. P.E. Kolenbrander, R.J. Palmer, S. Periasamy, N.S. Jakubovics, Oral multispecies biofilm development and the key role of cell-cell distance., Nat. Rev. Microbiol. 8 (2010) 471-80.

4. M. Kuboniwa, G.D. Tribble, C.E. James, A.O. Kilic, L. Tao, M.C. Herzberg, S. Shizukuishi, R.J. Lamont, Streptococcus gordonii utilizes several distinct gene functions to recruit Porphyromonas gingivalis into a mixed community, Mol. Microbiol. 60 (2006) 121-139.

5. C.P.J.M. Brouwer, M. Rahman, M.M. Welling, Discovery and development of a synthetic peptide derived from lactoferrin for clinical use., Peptides. 
32 (2011) 1953-63.

6. A. Ahmed, J. V. Rushworth, N.A. Hirst, P.A. Millner, Biosensors for whole-cell bacterial detection, Clin. Microbiol. Rev. 27 (2014) 631-646.

7. R.R. Silva, K.Y.P.S. Avelino, K.L. Ribeiro, O.L. Franco, M.D.L. Oliveira, C.A.S. Andrade, Optical and dielectric sensors based on antimicrobial peptides for microorganism diagnosis., Front. Microbiol. 5 (2014) 443.

8. M. Zasloff, Antimicrobial peptides of multicellular organisms, 415 (2002) 389-395.

9. A. Bratov, N. Abramova, J. Ramón-Azcón, A. Merlos, F. Sánchez-Baeza, M.-P. Marco, C. Domínguez, Characterisation of the interdigitated electrode array with tantalum silicide electrodes separated by insulating barriers, Electrochem. Commun. 10 (2008) 1621-1624.

10. A. Bratov, N. Abramova, M.P. Marco, F. Sanchez-Baeza, Three-Dimensional Interdigitated Electrode Array as a Tool for Surface Reactions Registration, Electroanalysis. 24 (2012) 69-75.

11. M. Godoy-gallardo, C. Mas-moruno, M.C. Fernández-calderón, C. Pérez-giraldo, Acta Biomaterialia Covalent immobilization of hLf1-11 peptide on a titanium surface reduces bacterial adhesion and biofilm formation, ACTA Biomater. (2014).

12. H.H. Huang, T.H. Lee, Electrochemical impedance spectroscopy study of Ti-6Al-4V alloy in artificial saliva with fluoride and/or bovine albumin, Dent. Mater. 21 (2005) 749-755.

13. C.E.B. Marino, L.H. Mascaro, EIS characterization of a Ti-dental implant in artificial saliva media: Dissolution process of the oxide barrier, J. Electroanal. Chem. 568 (2004) 115-120.

14. M. Zhu, M.Z. Lerum, W. Chen, How to prepare reproducible, homogeneous, and hydrolytically stable aminosilane-derived layers on silica., Langmuir. 28 (2012) 416-23.

15. X. Chen, P. Sevilla, C. Aparicio, Surface biofunctionalization by covalent co-immobilization of oligopeptides, Colloids Surfaces B Biointerfaces. 107 (2013) 189-197.

16. A. Bratov, N. Abramova, Response of a microcapillary impedimetric transducer to changes in surface conductance at liquid/solid interface, J. Colloid Interface Sci. 403 (2013) 151-156.

17. A. Bratov, J. Ramón-Azcón, N. Abramova, A. Merlos, J. Adrian, F. Sánchez-Baeza, M.-P. Marco, C. Domínguez, Three-dimensional interdigitated electrode array as a transducer for labelfree biosensors., Biosens. Bioelectron. 24 (2008) 729-35.

18. A. Shrivastava, V. Gupta, Methods for the determination of limit of detection and limit of quantitation of the analytical methods, Chronicles Young Sci. 2 (2011) 21-25.

19. P.K. Basu, D. Indukuri, S. Keshavan, V. Navratna, S.R.K. Vanjari, S. Raghavan, N. Bhat, Graphene based E. coli sensor on flexible acetate sheet, Sensors Actuators B Chem. 190 (2014) 342-347.

20. M.S. Mannoor, H. Tao, J.D. Clayton, A. Sengupta, D.L. Kaplan, R.R. Naik, N. Verma, F.G. Omenetto, M.C. McAlpine, Graphene-based wireless bacteria detection on tooth enamel., Nat. Commun. 3 (2012) 763. 